\title{
When the voluntary mind meets the irresistible event: Stimulus-response correspondence effects on task selection during voluntary task switching
}

\author{
Poyu Chen • Shulan Hsieh
}

Published online: 2 May 2013

(C) Psychonomic Society, Inc. 2013

\begin{abstract}
In the present study, we investigated how task selection is biased by inherent stimulus characteristics in the voluntary task-switching paradigm. We used digits as the task stimuli, since they may automatically induce spatially horizontal representations of numbers. Specifically, we examined whether an irrelevant spatial representation of a number coincides with its associated response codes and whether such a stimulus-response (S-R) correspondence effect biases task selection for a digit. Participants were randomly assigned to one of two different action code layout conditions: Two numerical tasks were arranged as inner/outer in the horizontal layout condition or as upper/down in the vertical layout condition. Participants in the horizontal layout condition were more likely to choose a task when the task's action code and the digit's spatial representation corresponded, as compared with when they did not. On the other hand, no selection bias was observed in the vertical layout condition, since there was no overlapping spatial representation between the stimulus and response. The present study extends previous findings by considering the influence of the stimulus-driven effect on task selection with regard to the $\mathrm{S}-\mathrm{R}$ correspondence effect.
\end{abstract}

Keywords Task selection - Voluntary task switching · Stimulus-response correspondence effect $\cdot$ Theory of event coding

In the laboratory environment, researchers often study action control by arbitrarily assigning a response to a stimulus

\section{P. Chen}

Department of Psychology, National Chung Cheng University,

Chiayi, Taiwan

\section{S. Hsieh $(\bowtie)$}

Department of Psychology, National Cheng Kung University,

No. 1, University Road, Tainan 701, Taiwan

e-mail: psyhs1@mail.ncku.edu.tw
(Donders, 1869). Success in executing the arbitrary stimulus-response (S-R) link should reflect an actor's intentional will. However, there is accumulating empirical evidence that a number of uninstructed S-R translations can be involuntarily induced upon processing of a stimulus (Hommel, 2000). To study voluntary action control, it is necessary to understand how a stimulus is presented and whether its representation will bind with a specific response to affect subsequent action planning (Prinz, 1997). In the present study, rather than assuming that $\mathrm{S}-\mathrm{R}$ translation is equivalent to the success of executing action control, we examined the intentional actions that people perform when inherent stimulus characteristics automatically interact with response representations.

Recently, the voluntary task-switching (VTS) paradigm has been proposed as a method for exploring the interplay between top-down and bottom-up processes in voluntary action control (Arrington, 2008; Demanet, Verbruggen, Liefooghe, \& Vandierendonck, 2010). Task selection is not immune to the influence of the characteristics of external stimuli, such as the repetition of a stimulus (Demanet et al., 2010; Mayr \& Bell, 2006), stimulus availability (Arrington, 2008), and the laterality of a stimulus representation (Arrington \& Rhodes, 2010), even though participants are explicitly instructed to select tasks as randomly and equally as possible. Moreover, Arrington, Weaver, and Paukner (2010) demonstrated that participants were more likely to categorize a stimulus as the same task as their initial choice regardless of whether the initial choice was derived from participants' previous selection (Arrington et al., 2010, Experiment 1) or was part of an experimental manipulation (Arrington et al., 2010, Experiment 2; see also Demanet et al., $2010)$ in a voluntary task choice context. The authors suggested that the encountered stimulus automatically triggered a previous $\mathrm{S}-\mathrm{R}$ binding that was stored in an event file such that the response in that event file became more available for biasing 
the subsequent task selection. Interestingly, their results revealed that there was extraexperimental $\mathrm{S}-\mathrm{R}$ binding other than the manipulated S-R binding that affected task selection; for example, participants tended to categorize aspirin as a "small" pill (size task) rather than as a "nonliving" pill (origin task) (Arrington et al., 2010, Experiment 1), or participants categorized more living stimuli, such as animals and plants, than nonliving stimuli as being the origin task (Arrington et al., 2010, Experiment 2) even though they were instructed to select the tasks equally. In other words, stimuli with inherent features seemed to bind with specific responses on the basis of past extraexperimental experiences. However, it is not easy to define the way in which the stimuli were represented. To our knowledge, research has not yet addressed whether and how an inherent stimulus representation binds with a specific response to bias task choice in the VTS paradigm.

Hommel, Musseler, Aschersleben, and Prinz (2001) proposed the theory of event coding (TEC) to explain how a stimulus binds with a response to affect action planning. The core argument of the TEC is that once the perception code (i.e., stimulus) and the action code (i.e., response) overlap in the same cognitive representation, known as the common code or event file, they are bidirectionally linked by virtue of similarity. The most prominent example of the bidirectional link between perception and action affecting action planning is the spatial S$\mathrm{R}$ correspondence effect (or the so-called Simon effect; Simon, 1969), in which reaction time (RT) is usually shorter and more accurate when a stimulus occurs in the same relative location as the response. Although the stimulus location is irrelevant to the task, information about the stimulus location still influences subsequent task performance with regard to the correspondence between the stimulus and response locations.

In the present study, our aim was to extend previous $\mathrm{S}-\mathrm{R}$ binding research by examining whether and how the bidirectional link between stimulus and response affects voluntary task selection, especially for inherent stimuli. The rationale was that the S-R correspondence effect may influence task selection if the stimulus and response representations overlap in the same common code. In contrast, a selection bias should not be observed if no common code exists. To this end, we used digits as task stimuli because they should automatically induce inherent spatial representations, including a form of quasispatial representation ${ }^{1}$ that is represented as a mental number line (Dehaene, Bossini, \& Giraux, 1993). In this representation, numerically smaller digits are processed faster with a left-lateralized response, and numerically larger digits are processed faster with a

\footnotetext{
${ }^{1}$ Although most studies address the S-R correspondence effect at the physical perception-action level, we believe that the mental quasispatial representation of digits can create similar perceptual information for us; that is, the S-R correspondence effect is present with an internal representation (Eimer, 1995) or is retrieved from memory (Hommel, 2002).
}

right-lateralized response, known as the spatial number association of response code (SNARC) effect. Importantly, we manipulated the action code by assigning two numerical tasks (i.e., magnitude and parity tasks) either horizontally represented (i.e., inner/outer) or vertically represented (i.e., upper/down) to overlap or not overlap with the horizontal mental number line on the basisiof finger-to-task assignment (Fig. 1b). To further examine how the S-R correspondence effect influences task choice, another between-subjects factor, the mapping group, was manipulated orthogonally to the layout condition. In the horizontal layout condition, participants were randomly assigned to one of two mapping groups in which the action code arrangements were opposites. In the outer-magnitude-inner-parity (oMiP) group, participants were asked to respond to the magnitude task with the "outer" action code (i.e., middle fingers) while responding to the parity task with the "inner" action code
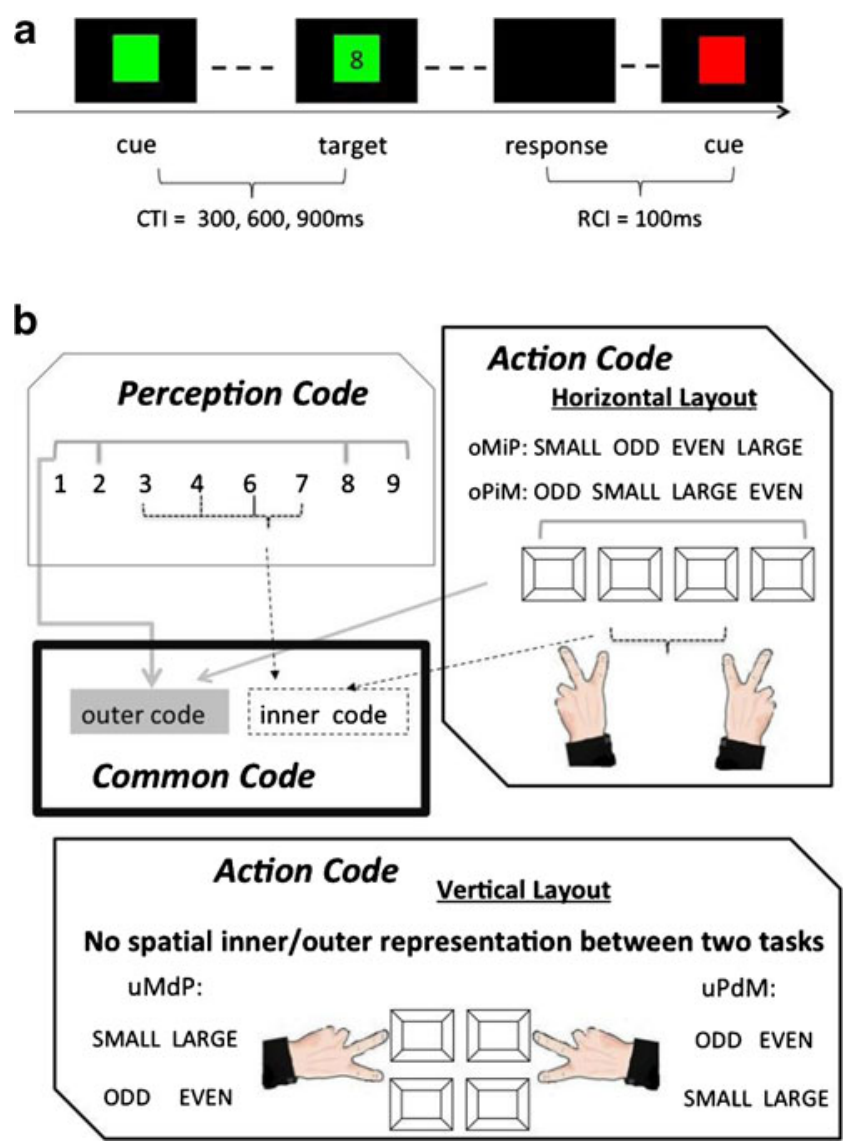

Fig. 1 Trial procedure and the action code arrangement. a The probabilities of cues of each color were equal, and the transition of color cue was maintained at 1:1. Target digits were presented randomly without successive repetition. b Metaphor of the common code representation between perception and action codes. In the horizontal layout condition, action codes were horizontally represented so that there were spatial inner and outer representations corresponding to the spatial representation induced by digit perception. In contrast, action codes were displayed as upper and down in the vertical layout condition. CTI, cue-target interval; RCI, response-cue interval 
(i.e., index fingers). This mapping was reversed in the outerparity-inner-magnitude (oPiM) group. In the vertical layout condition, there were also two mapping groups, such that, in the upper-magnitude-down-parity (uMdP) group, the upper action code (i.e., middle fingers) was mapped to the magnitude task and the lower action code (i.e., index fingers) was mapped to the parity task for a digit, and this mapping was reversed in the upper-parity-down-magnitude (uPdM) group. It is important to note that the finger-to-task assignments were exactly the same between the oMiP and uMdP groups and between the oPiM and uPdM groups. We adopted the different group names to emphasize the spatial representations evident in the horizontal and vertical layouts. Furthermore, given the orientation of the mental number line in the present study (see Hung, Hung, Tzeng, \& Wu, 2008), participants responded to small/large numbers with left-/right-side responses to avoid interfering with the digit representation. Additionally, the odd/even numbers were assigned to left-/right-side responses to avoid violating the linguistic markedness association of response codes effect (Berch, Foley, Hill, \& Ryan, 1999).

Given the overlapping inner/outer spatial features of the stimulus and response in the horizontal layout condition, we predicted that participants would categorize a digit more probably as the $\mathrm{S}-\mathrm{R}$ correspondent task than as the $\mathrm{S}-\mathrm{R}$ noncorrespondent task. That is, the oMiP group should categorize the quasispatial "outer" digit as the S-R correspondent "outer" magnitude task, while categorizing the quasispatial "inner" digit as the S-R correspondent "inner" parity task, given the integrated common code. The oPiM group should demonstrate the opposite selection pattern based on the opposite common code. In contrast, there should not be an opposite task selection bias between the uMdP and uPdM groups, because the upper/lower response representation does not correspond to the digit representation. Furthermore, given previous research demonstrating that preparation can minimize the stimulus-based priming effect (Arrington, 2008; Arrington \& Logan, 2005), we examined whether the S-R correspondence effect on selection bias could be modulated by advanced preparation.

However, one can argue that, with a single-key registration, the task selection bias, even if found in the horizontal layout condition, is a choice bias due not to $\mathrm{S}-\mathrm{R}$ correspondence per se but, rather, to the accessibility of the task - that is, a faster task response. Given the difficulty of disentangling task choice from measurements of task performance, an explicitly cued task-switching paradigm (CUE) with equal probabilities for the two switched tasks was also used. The rationale was that if the $\mathrm{S}-\mathrm{R}$ correspondence effect on task performance contributes to a biased task choice, then one should expect the $\mathrm{S}-\mathrm{R}$ correspondence RT effect in the VTS paradigm, but not in the equal task probability CUE paradigm. However, if task performance between the mapping groups is comparable for the VTS and CUE paradigms, one cannot argue that the selection bias is the by-product of task performance. Instead, task choice and execution might be modulated by two different cognitive systems (see also Arrington \& Yates, 2009).

\section{Method}

\section{Participants}

Forty-eight participants from National Chung Cheng University were randomly assigned to one of two layout conditions. In the horizontal layout condition, participants were separated into the oMiP and oPiM mapping groups. In the vertical layout condition, participants were separated into the uMdP and uPdM mapping groups. All participants signed an informed consent form and received monetary compensation for their participation.

\section{Materials and procedure}

The target stimuli included the digits 1 through 9 (excluding 5 ) presented at $0.6^{\circ}$. Stimuli were embedded in a square color cue (green or red) that had $1^{\circ}$ of visual angle. Two consecutive practice blocks of 64 trials were presented in order to familiarize participants with the S-R mappings of the magnitude task (greater or less than 5) and parity task (odd or even). After the practice blocks, participants received 10 blocks of 97 trials that included both the VTS and CUE paradigms in a counterbalanced order; that is, participants were randomly assigned to either the VTS-CUE or CUE-VTS order. On each trial, a target was presented after one of three randomized cuetarget intervals (CTIs), which were 300, 600, or $900 \mathrm{~ms}$ in length, was presented to encourage preparation for the upcoming task. Following the response and a 100-ms interval (response-cue interval; RCI), a cue for the next trial appeared (Fig. 1a). After each block, participants were given a short break. During the VTS paradigm, participants were told that the cues were uninformative and were asked to choose a task to perform as randomly and equally as possible. During the CUE paradigm, participants were asked to respond to the target digit according to the mapping rule between the cue color (red or green) and the task (the cue-color-task-mapping rule was counterbalanced between participants).

The response assignment was based on the finger-to-task assignment for both layout conditions. The critical difference between the two layout conditions was the spatial representation of the two tasks, since they were arranged as either inner/outer in the horizontal layout or upper/down in the vertical layout (Fig. 1b). Participants in both the oMiP and uMdP groups used their left and right middle fingers to categorize a digit as either smaller or larger than 5 , respectively, and used their left or right index fingers to indicate that a digit was either an odd or an even number, respectively. The finger-task mapping was reversed in the oPiM and 
uPdM groups, such that participants used their left and right middle fingers to categorize a digit as either odd or even, respectively and their left or right index fingers to indicate that a digit was either smaller or larger than 5 , respectively.

\section{Results}

After removing error trials, the following trials were also excluded from the analysis: trials with RTs less than $150 \mathrm{~ms}$ or greater than 3,000 ms, the first trials of each block, and the trials following error trials (data loss was $12.1 \%$ ). The proportion of error $(\mathrm{PE})$ data are reported in the supplemental material.

Task choice data

The main analysis of interest involved determining whether participants' choices were biased by the different action code layout manipulations. The response layout condition and mapping group were used as two between-subjects factors in an ANOVA, with digit and CTI as the withinsubjects factors. Given that the CTI factor neither reached significance nor interacted with other factors, we collapsed the three CTI levels to simplify the presentation of the following task choice analysis. The analysis of the CTI effect on task choice is presented in Appendix 1.

As is demonstrated in Table 1 and Fig. 2, the ANOVA revealed a significant three-way interaction between digit, layout, and mapping. A post hoc simple interaction test demonstrated that the task choice bias as a function of digit was significantly different between the two mapping groups in the horizontal layout condition, $F(7,308)=14.73, p<.01$, but not in the vertical layout condition, $F(7,308)=0.921, p=.50$. To further examine whether task choice for each digit was based on the S-R correspondence effect in the horizontal layout condition, a trend analysis (Keppel \& Wickens, 2004) was conducted separately for the two mapping groups. For the oMiP group, the trend analysis demonstrated significant linear, $F(1,77)=7.803, p<.05$, and quadratic, $F(1,77)=$

Table 1 Outcome of an ANOVA conducted on the selection probability for the parity task

\begin{tabular}{llll}
\hline Factors & df & F & $\eta 2$ \\
\hline Layout & 1,44 & 0.91 & 0.02 \\
Mapping & 1,44 & $5.17^{*}$ & 0.11 \\
Digit & 7,44 & $12.54^{*}$ & 0.22 \\
Layout $\times$ mapping & 1,44 & $9.26^{*}$ & 0.17 \\
Digit $\times$ layout & 7,44 & 1.48 & 0.03 \\
Digit $\times$ mapping & 7,44 & $9.79^{*}$ & 0.18 \\
Digit $\times$ layout $\times$ mapping & 7,44 & $5.86^{*}$ & 0.12 \\
\hline
\end{tabular}

$* p<.05$
$116.738, p<.05$, trends, such that participants tended to categorize a "quasispatial inner" digit as a parity task (S$\mathrm{R}$ correspondent) and a "quasispatial outer" digit as a magnitude task (S-R correspondent). On the contrary, significant quadratic, $F(1,77)=4.024, p<.05$, and cubic, $F(1,77)=$ $19.302, p<.05$, trends were observed in the oPiM group, such that participants tended to categorize the "quasispatial inner" digit as a magnitude task (S-R correspondent) and the "quasispatial outer" as a parity task (S-R correspondent). There was an unexpected effect in that the digit 9 was categorized as a magnitude task despite it being an S-R noncorrespondent condition for the oPiM mapping group. ${ }^{2}$

With regard to possible carryover effects from the paradigm order, we treated the order effect (i.e., VTS-CUE vs. CUE-VTS) as another between-subjects factor. Given that the critical S-R correspondence effect between the two mapping groups did not interact with the order effect regardless of the response layout condition, we present the figure and ANOVA results in Appendix 2.

\section{Task RT data}

We examined whether S-R correspondence was reflected in both the magnitude and parity RTs for the VTS context. Furthermore, we compared the VTS RTs with those from the CUE paradigm to examine whether the differing selection biases between the two mapping groups were a result of task performance. A 2 (layout) $\times 2$ (mapping) $\times 3(\mathrm{CTI}) \times 2$ (paradigm) $\times 8$ (digit) ANOVA was performed separately for the magnitude and parity RTs. The results revealed that the main effect of CTI reached significance for both the magnitude and parity RTs, which indicates that there was a general preparation effect on task performance (see Appendix 1, Fig. 5 and Table 4). In addition, the CTI effect interacted with the paradigm used for the parity RTs. A post hoc simple effect analysis revealed that the CTI effect was larger for the VTS paradigm, $F(2,176)=93.93, p<.01$, than for the CUE paradigm, $F(2,176)=38.30, p<.01$. Given that the CTI factor did not interact with the main interest factors, the mapping and/or layout factors, for either the magnitude or the parity RTs, we collapsed the three CTI levels to simplify presentation and to correspond with the task choice data (see Fig. 3 and Table 2).

\footnotetext{
${ }^{2}$ Regarding the strong magnitude bias for the digit 9, one might suggest that the magnitude bias on extreme digits originates from the SNARC effect, in which there is a left/right response selection advantage for extremely small/large digits in a free task choice context (Daar $\&$ Pratt, 2008). However, the lack of a magnitude bias (small) on digit 1 , regardless of the action code arrangement in the present study, may argue against the SNARC argument. The unique association between the digit 9 and a magnitude bias remains open for future research to examine.
} 
Fig. 2 Probability of selecting the parity task as a function of digits and separated by the two stimulus-response mappings in both horizontal and vertical layout conditions. Error bars indicate the standard errors. oMiP, outer-magnitude-innerparity; oPiM, outer-parityinner-magnitude; uMdP, uppermagnitude-down-parity; uPdM, upper-parity-downmagnitude

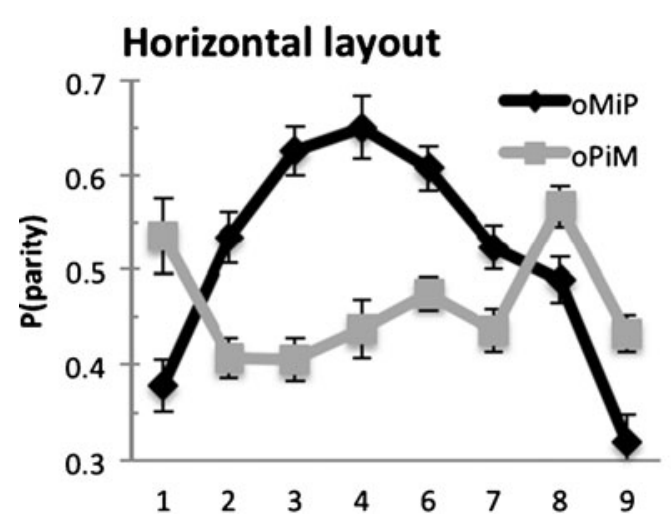

\section{Magnitude RT}

In general, participants took more time in the VTS paradigm than in the CUE paradigm. The digit main effect revealed that there was a significant distance effect such that RTs were shorter when the difference between the target digit and reference digit (5) was large. An S-R correspondence effect on magnitude RTs was not observed for either action code arrangement in either paradigm.

Regarding the significant two-way interaction between paradigm and digit, a post hoc simple effect test revealed that the digit effect was significant in the VTS paradigm, $F(7,154)=$ $14.582, p<.01$, and the CUE paradigm, $F(7,154)=12.416, p$ $<.01$. Moreover, a post hoc simple interaction effect test on the three-way interaction (digit $\times$ layout $\times$ mapping) suggested that the significant interaction between mapping group and digit was present in the horizontal layout condition, $F(7,308)=5.91$, $p<.01$, but not in the vertical layout condition, $F(7,308)=1.20$, $p=.37$. A post hoc simple effect test for the horizontal layout condition showed that the digit effect was larger for the oMiP mapping group, $F(7,154)=19.771, p<.01$, than for the oPiM group, $F(7,154)=5.335, p<.05$, which suggests that the location of the magnitude task coinciding with the outer extreme digits (i.e., the oMiP mapping groups in both paradigms) enlarged the distance effect. Critically, the null four-way interaction effect showed that the RTs between the mapping groups in the different layout conditions in the VTS paradigm were equivalent to those in the CUE paradigm, which suggests that the S-R correspondence effect on task choice in VTS was not a by-product of task performance.

\section{Parity RT}

Participants took more time to complete the task in the VTS paradigm than in the CUE paradigm. The digit main effect of
Fig. 3 Mean parity reaction time (RT) and magnitude RT in the two S-R mappings under the voluntary task-switching (VTS) and cued task-switching (CUE) paradigms as a function of digits. Error bars indicate the standard errors. oMiP, outermagnitude-inner-parity; uMdP, upper-magnitude-down-parity; oPiM, outer-parity-innermagnitude; uPdM, upperparity-down-magnitude

\section{Horizontal layout}
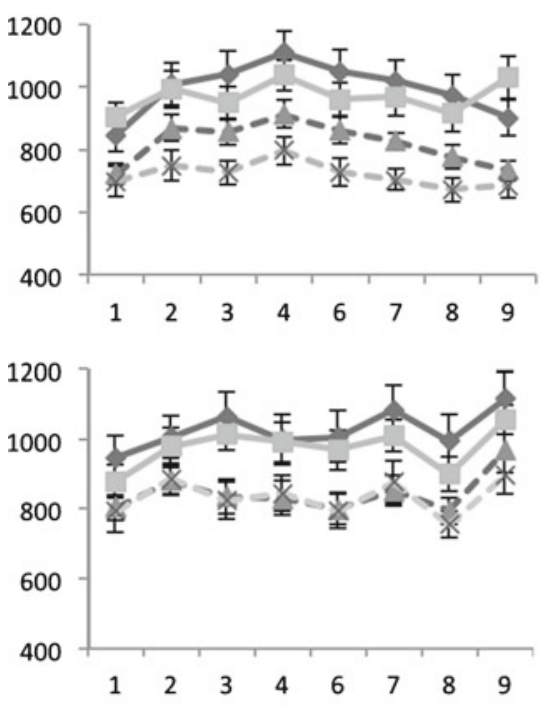

\section{Vertical layout}
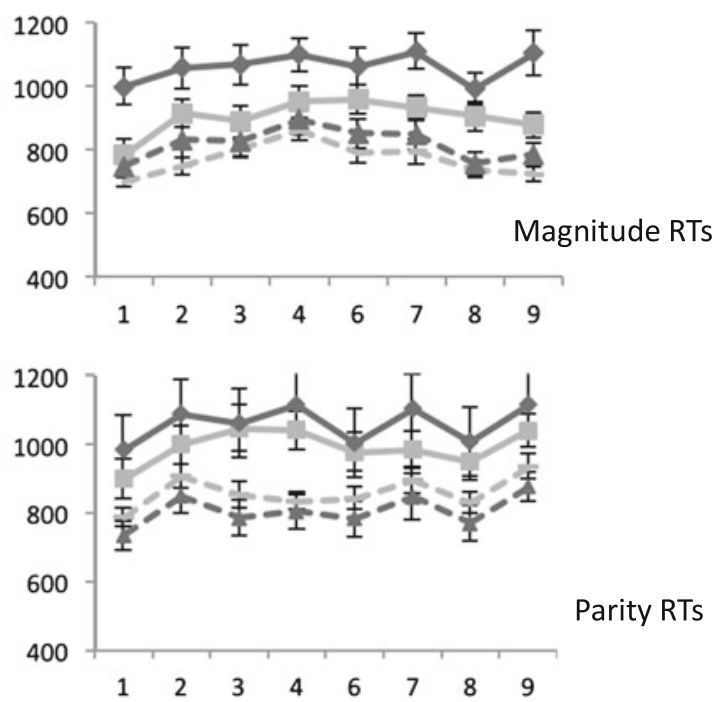
Table 2 Outcome of ANOVAs conducted on task performances in the magnitude and parity tasks

\begin{tabular}{|c|c|c|c|c|c|}
\hline \multirow[b]{2}{*}{ Factors } & \multirow[b]{2}{*}{ df } & \multicolumn{2}{|c|}{ Magnitude RT } & \multicolumn{2}{|c|}{ Parity RT } \\
\hline & & $\mathrm{F}$ & $\eta 2$ & $\mathrm{~F}$ & $\eta 2$ \\
\hline Layout & 1,44 & 0.03 & 0.00 & 0.07 & 0.00 \\
\hline Mapping & 1,44 & 3.05 & 0.07 & 0.03 & 0.00 \\
\hline Paradigm & 1,44 & $76.24 *$ & 0.63 & $59.92 *$ & 0.58 \\
\hline Digit & 7,44 & $33.89^{*}$ & 0.44 & $36.93^{*}$ & 0.46 \\
\hline Layout $\times$ mapping & 1,44 & 0.03 & 0.00 & 0.05 & 0.00 \\
\hline Paradigm $\times$ layout & 1,44 & 2.40 & 0.05 & 0.05 & 0.00 \\
\hline Paradigm $\times$ mapping & 1,44 & 0.05 & 0.00 & 2.18 & 0.05 \\
\hline $\begin{array}{l}\text { Paradigm } \times \text { layout } \times \\
\text { mapping }\end{array}$ & 1,44 & 2.80 & 0.06 & 0.23 & 0.01 \\
\hline Digit $\times$ layout & 7,44 & 1.30 & 0.029 & 1.41 & 0.03 \\
\hline Digit $\times$ mapping & 7,44 & $2.06^{*}$ & 0.045 & 0.71 & 0.02 \\
\hline $\begin{array}{l}\text { Digit } \times \text { layout } \times \\
\text { mapping }\end{array}$ & 7,44 & $5.09 *$ & 0.104 & 1.03 & 0.02 \\
\hline Paradigm $\times$ digit & 7,44 & $2.91^{*}$ & 0.062 & $4.28 *$ & 0.09 \\
\hline $\begin{array}{l}\text { Paradigm } \\
\text { layout }\end{array}$ & 7,44 & 0.63 & 0.014 & 1.60 & 0.04 \\
\hline $\begin{array}{l}\text { Paradigm } \times \text { digit } \times \\
\text { mapping }\end{array}$ & 7,44 & 0.82 & 0.018 & 0.59 & 0.01 \\
\hline $\begin{array}{l}\text { Paradigm } \times \text { digit } \times \\
\text { layout } \times \text { mapping }\end{array}$ & 7,44 & 1.46 & 0.032 & 0.27 & 0.01 \\
\hline
\end{tabular}

$* p<.05$

parity RTs revealed a response congruency-like effect, ${ }^{3}$ in which responses were faster to both the relevant and irrelevant tasks when they were assigned to the same response side. An S-R correspondence effect on parity RTs was not observed for either action code arrangement in either paradigm.

Moreover, a post hoc simple effect test on the two-way interaction between paradigm and digit demonstrated that the response congruency-like effect was significant in both the VTS paradigm, $F(7,616)=22.56, p<.01$, and the CUE paradigm, $F(7,616)=22.07, p<.01$. Again, the null four-way interaction effect ruled out the possibility that task choice bias was the by-product of task performance.

\section{Discussion}

In the present study, we extended previous findings regarding stored S-R bindings in event files (Arrington et al., 2010) by considering whether and how the inherent stimulus binds with specific responses to affect voluntary task choices. Given the inherent spatial features embedded in digits, we demonstrated how the response representation determines the task choice

\footnotetext{
$\overline{3}$ The response congruency effect is not restricted to the physically "exact" same unit. Instead, an abstract response meaning is enough to induce the response congruency effect (Gade \& Koch, 2007). Therefore, the response congruency-like effect should be present even when the response was univalent in the present design.
}

with regard to the overlapping S-R representation. That is, when the response representation of the two tasks overlapped with the digit representation as an inner/outer common code in the horizontal layout condition, participants tended to categorize a digit as the S-R correspondent task more often than the S-R noncorrespondent task. In contrast, participants did not show a task choice bias between the mapping groups in the vertical layout condition, since the response representation of the upper/down action code arrangement did not correspond to the digit representation. Furthermore, the S-R correspondence effect on task choice bias was not the result of task performance. If task selection is independent of execution, this would support the argument that task selection and task performance involve two different cognitive control mechanisms (Arrington \& Yates, 2009; see also Orr \& Weissman, 2011).

Arrington and Logan (2005) proposed the following two competing heuristics for choosing the task: the top-down representativeness heuristic and the bottom-up availability heuristic. According to Arrington and Logan, the representativeness heuristic requires comparing a recent task choice sequence in working memory with a random sequence, which is an internal control process that requires time to perform. Related to Arrington and Logan's formulation of the representativeness heuristic, Mayr and Bell (2006) suggested that random task choices could be achieved by treating each task choice as a discrete event - that is, a mental coin flip. In addition, there is a recent model from Vandierendnock and his colleagues (Vandierendonck, Demanet, Liefooghe, \& Verbruggen, 2012) that posits that participants select tasks by retrieving a short sequence (e.g., the subset for sequences of length 4) from the long-term memory on the basis of the task names. ${ }^{4}$ On the other hand, the availability heuristic is in charge of task choice when preparation time is limited, whereas the representativeness heuristic is responsible for task choice when preparation time is longer, as demonstrated in Arrington's (2008) stimulus availability manipulation. However, in the present study, response retrieval depended primarily on the S-R correspondence regardless of advance preparation, which was similar to the finding that response availability was immune to the top-down modulations (Arrington \& Rhodes, 2010). It seems that the present S-R correspondence acts more like a prepared reflex representation (Hommel, 2000), in which a cognitive process is triggered even when it is not intended. According to the dual-route model, both intentional and automatic routes combine to form a complex SR translation process (Kornblum, Hasbroucq, \& Osman, 1990). Once the automatic route has been activated by an overlapping S-R representation, the intentional-based control becomes less involved in the action control.

As Haggard's (2008) review of voluntary actions suggests, an experimental approach to studying voluntary

\footnotetext{
${ }^{4}$ The authors would like to thank the reviewer Joseph Orr for providing this important information.
} 
action depends on the amount of resistance provided by stimulus-driven effects. In fact, it is rare that people make voluntary actions in an environmental "vacuum." When studying voluntary action control, it is important to avoid treating the stimulus as a mere action precursor. Instead, it is important to consider the functional link between perception and action and how it could modulate action planning and influence task selection (Hommel et al., 2001).

Author Note The authors would like to thank the National Science Council of the Republic of China, Taiwan for financially supporting this research (Contract No. NSC98-2410-H-006-113-MY3). Also, the authors are indebted to Joseph Orr, two anonymous reviewers, and the action editor, Mei-Ching Lien, for their valuable and constructive comments on earlier versions of the manuscript.

\section{Appendix 1}

The CTI effects on task choice are plotted in Fig. 4, and the related ANOVA is presented in Table 3. The performances of magnitude and parity and their related ANOVAs are presented separately in Figure 5 and Table 4.
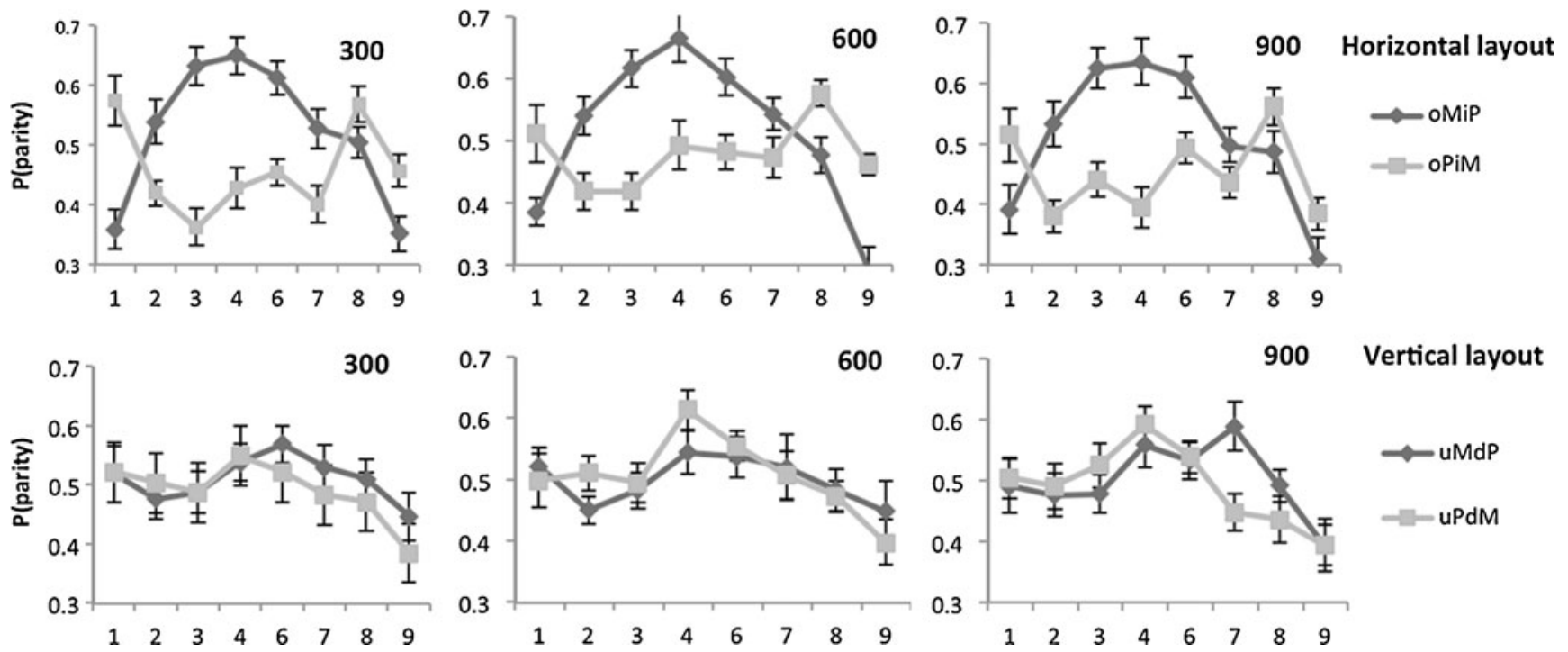

Fig. 4 Probability of parity task as a function of digits and separated by the two S-R mappings across three CTIs. Error bars indicate the standard errors

Table 3 Outcome of an ANOVA conducted on the probability of selecting the parity task, with cue-target interval (CTI) as an additional factor

\begin{tabular}{llll}
\hline Factors & df & F & $\eta 2$ \\
\hline Layout & 1,44 & 1.02 & 0.023 \\
Mapping & 1,44 & $5.10^{*}$ & 0.10 \\
CTI & 2,88 & 2.29 & 0.05 \\
digit & 7,308 & $12.50^{*}$ & 0.22 \\
Layout $\times$ Mapping & 1,44 & $8.93^{*}$ & 0.17 \\
CTI $\times$ Layout & 2,88 & 0.51 & 0.01 \\
CTI $\times$ Mapping & 2,88 & 0.05 & 0.00 \\
CTI $\times$ Layout $\times$ Mapping & 2,88 & 2.01 & 0.03 \\
digit $\times$ Layout & 7,308 & 1.51 & 0.03 \\
digit $\times$ Mapping & 7,308 & $9.58^{*}$ & 0.18 \\
digit $\times$ Layout $\times$ Mapping & 7,308 & $5.75^{*}$ & 0.12 \\
CTI $\times$ digit & 14,616 & 1.26 & 0.03 \\
CTI $\times$ digit $\times$ Layout & 14,616 & 0.67 & 0.02 \\
CTI $\times$ digit $\times$ Mapping & 14,616 & 1.15 & 0.03 \\
CTI $\times$ digit $\times$ Layout $\times$ Mapping & 14,616 & 1.12 & 0.03 \\
\hline
\end{tabular}

$* p<.05$ 

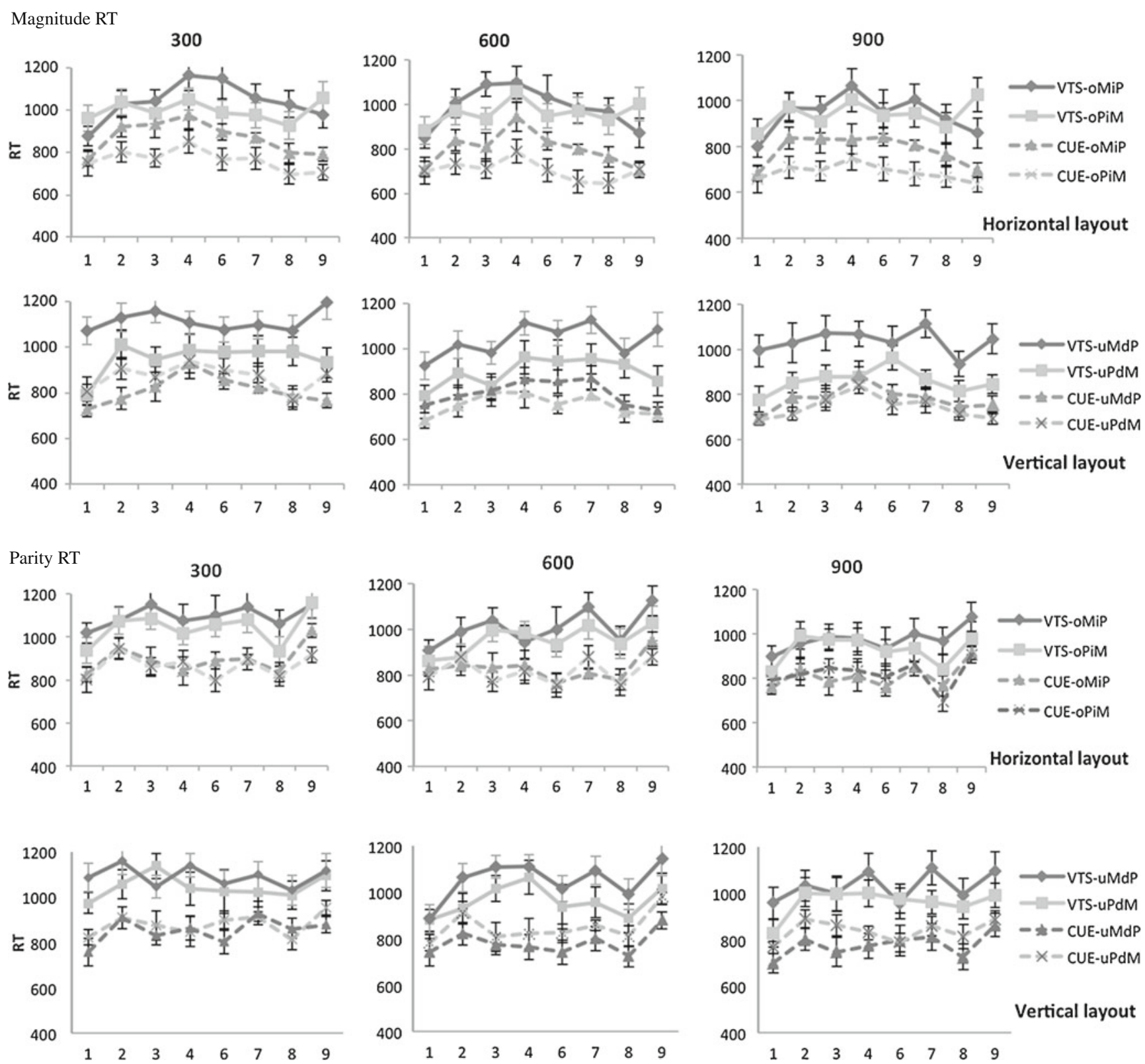

Fig. 5 Mean magnitude and parity reaction times (RTs) in the two S-R mappings with cue-target interval (CTI) as an additional factor under the VTS and CUE paradigms, as a function of digit. Error bars indicate the standard errors. VTS, voluntary task switching; CUE, cued task switching; oMiP, outer-magnitude-inner-parity; oPiM, outer-parityinner-magnitude; uMdP, upper-magnitude-down-parity; uPdM, upper-parity-down-magnitude 
Table 4 Outcome of ANOVAs conducted on task performances in the magnitude and parity tasks with cue-target interval (CTI) as an additional factor

\begin{tabular}{|c|c|c|c|c|c|}
\hline \multirow[b]{2}{*}{ Factors } & \multirow[b]{2}{*}{ df } & \multicolumn{2}{|c|}{ Magnitude RT } & \multicolumn{2}{|c|}{ Parity RT } \\
\hline & & $\mathrm{F}$ & $\eta 2$ & $\mathrm{~F}$ & $\eta 2$ \\
\hline Layout & 1,44 & 0.03 & 0.00 & 0.07 & 0.002 \\
\hline Mapping & 1,44 & 3.10 & 0.07 & 0.04 & 0.001 \\
\hline Layout $\times$ Mapping & 1,44 & 0.04 & 0.00 & 0.23 & 0.005 \\
\hline Paradigm & 1,44 & $76.59 *$ & 0.64 & $58.82 *$ & 0.57 \\
\hline paradigm $\times$ Layout & 1,44 & 2.22 & 0.05 & 0.03 & 0.00 \\
\hline paradigm $\times$ Mapping & 1,44 & 0.05 & 0.00 & 1.93 & 0.04 \\
\hline paradigm $\times$ Layout $\times$ Mapping & 1,44 & 2.78 & 0.06 & 0.23 & 0.01 \\
\hline CTI & 2,88 & $98.54 *$ & 0.69 & $124.93 *$ & 0.74 \\
\hline CTI $\times$ Layout & 2,88 & 0.46 & 0.63 & 1.35 & 0.03 \\
\hline CTI $\times$ Mapping & 2,88 & 1.01 & 0.02 & 2.62 & 0.06 \\
\hline CTI $\times$ Layout $\times$ Mapping & 2,88 & 0.21 & 0.01 & 0.07 & 0.00 \\
\hline digit & 7,308 & $33.44 *$ & 0.43 & $37.03 *$ & 0.46 \\
\hline digit $\times$ Layout & 7,308 & 1.41 & 0.03 & 1.14 & 0.03 \\
\hline digit $\times$ Mapping & 7,308 & 2.08 & 0.05 & 0.75 & 0.02 \\
\hline digit $\times$ Layout $\times$ Mapping & 7,308 & 5.28 & 0.11 & 0.94 & 0.02 \\
\hline paradigm $\times$ CTI & 2,88 & 0.63 & 0.01 & $6.38^{*}$ & 0.13 \\
\hline paradigm $\times \mathrm{CTI} \times$ Layout & 2,88 & 0.61 & 0.01 & 0.71 & 0.02 \\
\hline paradigm $\times \mathrm{CTI} \times$ Mapping & 2,88 & 0.04 & 0.00 & 1.14 & 0.03 \\
\hline paradigm $\times \mathrm{CTI} \times$ Layout $\times$ Mapping & 2,88 & 1.30 & 0.03 & 0.88 & 0.02 \\
\hline paradigm $\times$ digit & 7,308 & $3.14^{*}$ & 0.07 & $4.31 *$ & 0.09 \\
\hline paradigm $\times$ digit $\times$ Layout & 7,308 & 0.63 & 0.01 & 1.45 & 0.03 \\
\hline paradigm $\times$ digit $\times$ Mapping & 7,308 & 0.83 & 0.02 & 0.41 & 0.01 \\
\hline paradigm $\times$ digit $\times$ Layout $\times$ Mapping & 7,308 & 1.45 & 0.03 & 0.52 & 0.01 \\
\hline $\mathrm{CTI} \times$ digit & 14,616 & 0.46 & 0.01 & 1.29 & 0.03 \\
\hline $\mathrm{CTI} \times$ digit $\times$ Layout & 14,616 & 1.31 & 0.03 & 0.72 & 0.02 \\
\hline $\mathrm{CTI} \times$ digit $\times$ Mapping & 14,616 & 0.62 & 0.01 & 1.32 & 0.03 \\
\hline CTI $\times$ digit $\times$ Layout $\times$ Mapping & 14,616 & 0.85 & 0.02 & 1.12 & 0.03 \\
\hline paradigm $\times \mathrm{CTI} \times$ digit & 14,616 & 0.67 & 0.02 & 1.75 & 0.04 \\
\hline paradigm $\times \mathrm{CTI} \times$ digit $\times$ Layout & 14,616 & 1.51 & 0.10 & 0.80 & 0.02 \\
\hline paradigm $\times \mathrm{CTI} \times$ digit $\times$ Mapping & 14,616 & 1.46 & 0.03 & 1.39 & 0.03 \\
\hline paradigm $\times$ CTI $\times$ digit $\times$ Layout $\times$ Mapping & 14,616 & 0.64 & 0.01 & 1.21 & 0.04 \\
\hline
\end{tabular}




\section{Appendix 2}

The order effect was treated as an additional betweensubjects order factor. Figure 6 depicts the mean probability for the parity task separated by the two orders, and the related ANOVA is presented in Table 5.
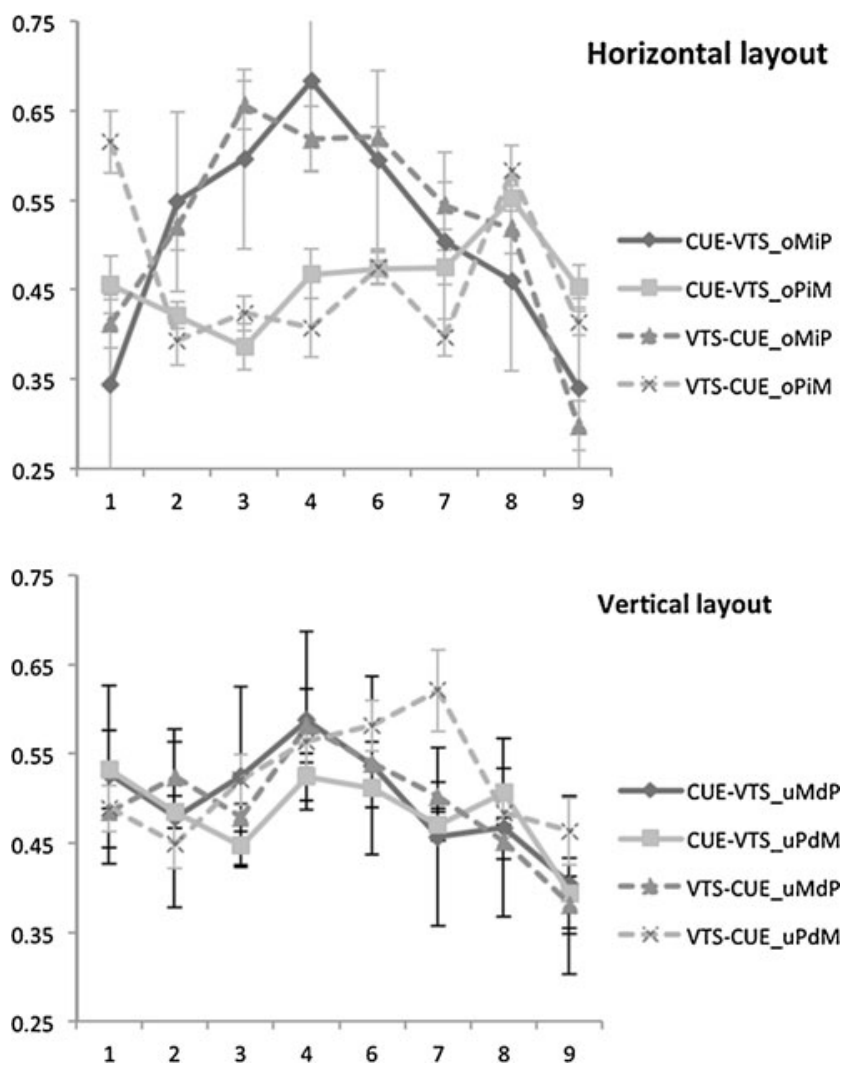

Fig. 6 Probability of parity task as a function of digits and separated by the two stimulus-response mappings with order effect as an additional between-subjects factor. Error bars indicate the standard errors. CUE, cued task switching; VTS, voluntary task switching; oMiP, outer-magnitude-inner-parity; oPiM, outer-parity-inner-magnitude; upper-magnitude-down-parity; upper-parity-down-magnitude
Table 5 Outcome of an ANOVA conducted on the probability of selecting the parity task, with order effect as an additional betweensubjects factor

\begin{tabular}{llll}
\hline Factors & df & F & $\eta 2$ \\
\hline Layout & 1,40 & 0.91 & 0.02 \\
Mapping & 1,40 & $5.16^{*}$ & 0.11 \\
Order & 1,40 & 1.49 & 0.04 \\
Digit & 7,280 & $12.75^{*}$ & 0.24 \\
Layout $\times$ mapping & 1,40 & $9.26^{*}$ & 0.19 \\
Order $\times$ layout & 1,40 & 0.14 & 0.00 \\
Order $\times$ mapping & 1,40 & 0.56 & 0.01 \\
Order $\times$ layout $\times$ mapping & 1,40 & 1.78 & 0.04 \\
Digit $\times$ layout & 7,280 & 1.50 & 0.04 \\
Digit $\times$ mapping & 7,280 & $9.96^{*}$ & 0.20 \\
Digit $\times$ layout $\times$ mapping & 7,280 & $5.96^{*}$ & 0.13 \\
Order $\times$ digit & 7,280 & 0.78 & 0.02 \\
Order $\times$ digit $\times$ layout & 7,280 & 2.03 & 0.06 \\
Order $\times$ digit $\times$ mapping & 7,280 & 0.37 & 0.01 \\
Order $\times$ digit $\times$ layout $\times$ mapping & 7,280 & 0.97 & 0.02 \\
\hline
\end{tabular}

$* p<.05$

\section{References}

Arrington, C. M. (2008). The effect of stimulus availability on task choice in voluntary task switching. Memory \& Cognition, 36, 991-997.

Arrington, C. M., \& Logan, G. D. (2005). Voluntary task switching: chasing the elusive homunculus. Journal of Experimental Psychology: Learning, Memory, \& Cognition, 31, 683-702.

Arrington, C. M., \& Rhodes, K. M. (2010). Perceptual asymmetries influence task choice: the effect of lateralised presentation of hierarchical stimuli. Laterality, 15, 501-513.

Arrington, C. M., Weaver, S. M., \& Paukner, S. (2010). Stimulus-based priming of task choice during voluntary task switching. Journal of Experimental Psychology: Learning Memory, \& Cognition, 36, 1060-1067.

Arrington, C. M., \& Yates, M. M. (2009). The role of attentional networks in voluntary task switching. Psychonomic Bulletin \& Review, 16, 660-665. 
Berch, D. B., Foley, E. J., Hill, R. J., \& Ryan, P. M. (1999). Extracting parity and magnitude from Arabic numerals: developmental changes in number processing and mental representation. Journal of Experimental Child Psychology, 74, 286-308.

Daar, M., \& Pratt, J. (2008). Digits affect actions: the SNARC effect and response selection. Cortex, 44, 400-405.

Dehaene, S., Bossini, S., \& Giraux, P. (1993). The mental representation of parity and number magnitude. Journal of Experimental Psychology: General, 122, 371-396.

Demanet, J., Verbruggen, F., Liefooghe, B., \& Vandierendonck, A. (2010). Voluntary task switching under load: Contribution of top-down and bottom-up factors in goal-directed behavior. Psychonomic Bulletin \& Review, 3, 387-393.

Donders, F. (1869). On the speed of mental process. Acta Psychologia, 30, 412-431.

Eimer, M. (1995). Stimulus-response compatibility and automatic response activation: evidence from psychophysiological studies. Journal of Experimental Psychology. Human Perception, \& Performance, 21, 837-854.

Gade, M., \& Koch, I. (2007). The influence of overlapping response sets on task inhibition. Memory \& Cognition, 35, 603-609.

Haggard, P. (2008). Human volition: towards a neuroscience of will. Nature Reviews Neuroscience, 9, 934-946.

Hommel, B. (2000). The prepared reflex: Automaticity and control in stimulus-response translation. In S. Monsell \& J. Driver (Eds.), Control of cognitive processes: Attention and performance XVIII (pp. 247-273). MIT Press: Cambridge, MA.

Hommel, B. (2002). Responding to object files: automatic integration of spatial information revealed by stimulus-response compatibility effects. Quarterly Journal of Experimental Psychology, 55A, 567-580.

Hommel, B., Musseler, J., Aschersleben, G., \& Prinz, W. (2001). The Theory of Event Coding (TEC): a framework for perception and action planning. Behavoral \& Brain Science, 24, 849-878. discussion 878-937.

Hung, Y. H., Hung, D. L., Tzeng, O. J., \& Wu, D. H. (2008). Flexible spatial mapping of different notations of numbers in Chinese readers. Cognition, 106, 1441-1450.

Keppel, G., \& Wickens, T. (2004). Design and analysis: a researcher's handbook (4th ed.). Upper Saddle River, New Jersey: Pearson Education, Inc.

Kornblum, S., Hasbroucq, T., \& Osman, A. (1990). Dimensional overlap: cognitive basis for stimulus-response compatibility-a model and taxonomy. Psychological Review, 97, 253-270.

Mayr, U., \& Bell, T. (2006). On how to be unpredictable: evidence from the voluntary task-switching paradigm. Psychological Science, 17, 774-780.

Orr, J. M., \& Weissman, D. H. (2011). Succumbing to bottom-up biases on task choice predicts increased switch costs in the voluntary task switching paradigm. Frontiers in Psychology, 2, 31.

Prinz, W. (1997). Why Donders has led us astray. In B. Hommel \& W. Prinz (Eds.), Theoretical issues in stimulus-response compatibility (pp. 247-267). Amsterdam, The Netherland: North-Holland.

Simon, J. R. (1969). Reactions toward the source of stimulation. Journal of Experimental Psychology, 81, 174-176.

Vandierendonck, A., Demanet, J., Liefooghe, B., \& Verbruggen, F. (2012). A chain-retrieval model for voluntary task switching. Cognitive Psychology, 65, 241-283. 